\title{
El Proyecto EPBE de la UDIMA, un impulso a la empleabilidad de los
}

\section{jóvenes europeos}

\section{Extracto:}

El European Personal Branding for Employment (EPBE) es un proyecto subvencionado por el Programa Erasmus+, concretamente en la Acción Clave 2: "Cooperación para la innovación y el intercambio de buenas prácticas».

\section{Sumario}

1. Introducción-justificación

\section{Objetivos del proyecto EPBE}

\section{Destinatarios y metodología}

\section{Productos y resultados}

5. Conclusiones

6. Valoración del impacto del proyecto

\section{Bibliografía}

La UDIMA finaliza este septiembre el Proyecto EPBE, que ha llevado a cabo en coordinación con otras instituciones, en concreto, con universidades, centros de formación y empresas de Italia, Grecia, Alemania, Chipre y España.

El objetivo del proyecto ha sido fomentar el empleo entre los jóvenes mediante la adquisición de competencias digitales.

Los destinatarios no son únicamente los jóvenes de la Unión Europea, los estudiantes de educación superior y formación profesional, sino también las personas demandantes de empleo, los docentes pertenecientes a las instituciones participantes y otros formadores interesados, así como cualquiera que desee mejorar sus referencias y aumentar su empleabilidad.

Para conseguir este objetivo, la UDIMA y el resto de instituciones participantes han utilizado innovadores métodos pedagógicos y han elaborado multitud de recursos educativos en abierto, entre los que se encuentran un curso de formación para los docentes y dos massive online open courses (MOOC) disponibles en la Plataforma UDEMY.

En el artículo se describen los recursos y resultados dentro del marco de dicho proyecto.

Palabras clave: marca personal, competencias digitales, búsqueda de empleo 4.0, empleabilidad, massive online open courses (MOOC), metodologías-pedagogías innovadoras.

\footnotetext{
1 S. Delgado Resino, miembro de la Unidad de Innovación Docente de la Universidad a Distancia de Madrid (UDIMA).

${ }^{2}$ A. Landeta Etxeberria, directora del Proyecto EPBE y del Instituto de l+D+i de la Universidad a Distancia de Madrid (UDIMA).

${ }^{3}$ S. N. Prieto Preboste, coordinadora de la Unidad de Innovación Docente de la Universidad a Distancia de Madrid (UDIMA).
}

Co-funded by the Erasmus+ Programme of the European Union
European Personal Branding for Employment (EPBE) es un proyecto subvencionado por el Programa Erasmus+, concretamente en la Acción Clave 2: «Cooperación para la innovación y el intercambio de buenas prácticas». 


\section{UDIMA's EPBE Project, a boost for the employability of young european people}

\section{Abstract:}

The European Personal Branding for Employment (EPBE) is a project subsidised by the Erasmus+ Programme, specifically in Key Action 2: "Cooperation for the innovation and exchange of good practices».

UDIMA concludes the EPBE Project this september, which was implemented in partnership with other institutions, in particular, universities, training centres and companies in Italy, Greece, Germany, Cyprus and Spain.

The aim of the project was to encourage employment among young people through the acquisition of digital skills.

The beneficiaries are not only young people from the European Union, students of higher education and professional training, but also jobseekers, teachers belonging to participating institutions and other interested trainers, including those who wanted to improve their references and increase their employability.

In order to achieve this objective, UDIMA and the other participating institutions have used innovative pedagogical methods and generated a large number of open educational resources, including a training course for teachers and two massive online open courses (MOOCs) available in UDEMY Platform.

The article describes the resources and results produced within the framwork of this project.

Keywords: personal branding, digital skills, job hunting 4.0, employability, massive online open courses (MOOCs), innovative pedagogical methods.

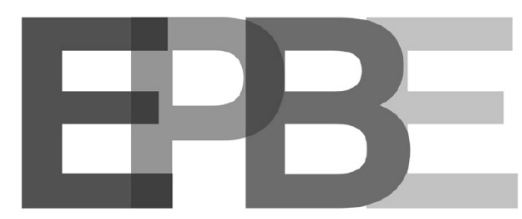

European Personal Branding for Employment

\section{INTRODUCCIÓN-JUSTIFI- CACIÓN}

Si dirigimos nuestra atención a las necesidades actuales de los jóvenes españoles y, por extensión, europeos, nuestro foco se centra, sobre todo, en el empleo. $Y$ es que el dinámico mercado laboral actual, el incremento general del nivel formativo de los estudiantes $y$ de la competencia entre demandantes de empleo y el desarrollo y el avance continuo de las tecnologías de la información y la comunicación (TIC) son variables que suman dificultades a los jóvenes que luchan cada día por conseguir su objetivo profesional.

En la actualidad, muchas instituciones de educación superior, como agentes formadores de los jóvenes y como promotoras de cambio, dedican gran parte de sus presupuestos y esfuerzos a hacer frente a esta problemática, desarrollando e implementando proyectos que favorecen la mejora de las competencias profesionales de los estudiantes. Como citan Barrenetxea y Cardona (2003), «en el actual entorno laboral cambiante, la información, la formación, la educación y el aprendizaje durante toda la vida son activos esenciales».

La UDIMA, como institución educativa de educación superior, sin dejar a un lado este propósito, se planteó hace dos años llevar a cabo un proyecto a nivel europeo en coordinación con otras instituciones para hacer frente a la brecha digital que aún hoy produce desigualdades en materia de empleo entre los jóvenes. El proyecto, denominado EPBE y subvencionado por el Programa Erasmus+ dentro de la Acción Clave 2, «Cooperación para la innovación y el intercambio de buenas prácticas», se apoya en el hecho de que, actualmente, el uso y el dominio de las competencias digitales son im- 
«La formación en competencias informacionales y digitales [...] debería ser una meta recurrente y permanente de todo el sistema educativo desde la educación infantil hasta la superior» (Área, 2010)

prescindibles para mejorar las referencias e incrementar la empleabilidad de las personas. Nos atreveríamos a decir, de hecho, que «la formación en competencias informacionales y digitales 0 , dicho de otra forma, la alfabetización de los individuos ante los múltiples lenguajes y códigos (textuales, icónicos, hipertextuales, audiovisuales, multimedia, etc.) que adopta la información debería ser una meta recurrente y permanente de todo el sistema educativo desde la educación infantil hasta la superior» (Área, 2010).

Este mismo autor expone los argumentos más destacables por los cuales la enseñanza universitaria debería promover esta alfabetización:

«1. La producción de conocimiento en todas las áreas del saber -científico, técnico, humanístico, artístico o social- en estas últimas décadas está en permanente crecimiento exponencial y es prácticamente inabarcable. [...]

2. Existen cada vez mayores y numerosas fuentes que almacenan, organizan y difunden información en formato de bibliotecas digitales, bases de datos, portales web, publicaciones electrónicas, blogs, redes sociales, etc. [...].

3. Las teorías pedagógicas y del aprendizaje señalan que el conocimiento debe ser construido por cada estudiante como un proceso experiencial, en interacción con otros sujetos y a través de la acción. [...].

4. Las formas de expresión y comunicación de las ideas, sentimientos, opiniones y conocimientos adoptan formas y lenguajes múl- tiples que se proyectan en textos escritos, en documentos audiovisuales o en archivos multimedia. [...].

5. Desde hace una década, los espacios virtuales están ganando mayor protagonismo en la enseñanza universitaria y configuran modalidades educativas conocidas como e-learning, docencia virtual, educación semipresencial o b-learning y similares. Esta incorporación de las TIC a la docencia universitaria requiere que tanto alumnado como profesorado dispongan del dominio y de las competencias del manejo de las herramientas de LMS (learning management system), así como de los distintos recursos que configuran la denominada Web 2.0».
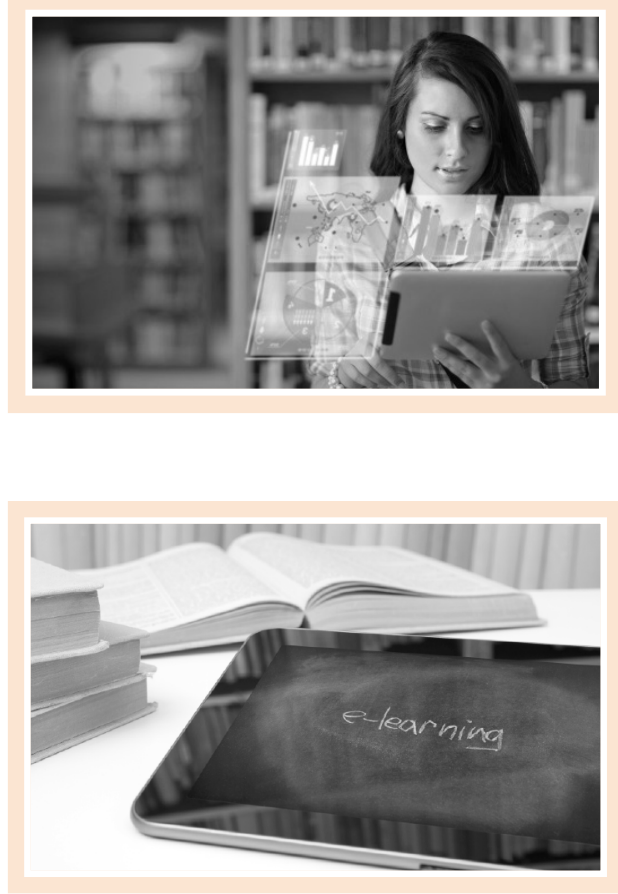

Encontramos total justificación al proyecto EPBE si echamos mano de las últimas políticas sobre empleo, asuntos sociales e inclusión promovidas por la Comisión Europea, y es que una de ellas, la iniciativa New 


\section{El proyecto EPBE nace con la misión de romper la brecha digital para aumentar la empleabilidad de los jóvenes europeos, mejorando sus competencias en búsqueda de empleo a través de la red y favoreciendo la creación de una marca personal online}

Skills for New Jobs ${ }^{4}$ (Nuevas Competencias para Nuevos Empleos), nació con el objeto de:

- Promover una mejor anticipación de las futuras necesidades competenciales.

- Mejorar la correspondencia entre las competencias y las necesidades del mercado laboral.

- Reducir la brecha entre los mundos de la educación y el trabajo.

Tal y como indica la web de la Comisión Europea, las medidas prácticas de New Skills for New Jobs incluyen, como uno de sus referentes, el marco europeo de competencias clave para el aprendizaje permanente, que define las ocho competencias que todo ciudadano debe poseer para prosperar en una sociedad del conocimiento. Una de ellas precisamente es la competencia digital, a la que define como aquella competencia que «entraña el uso seguro y crítico de las tecnologías de la sociedad de la información (TSI) para el trabajo, el ocio y la comunicación. Se sustenta en las competencias básicas en materia de TIC: el uso de ordenadores para obtener, evaluar, almacenar, producir, presentar e intercambiar información, y comunicarse y participar en redes de colaboración a través de internet» (Comisión Europea, 2006).

Actualmente, en el contexto de la sociedad de la información en el que vivimos, ser «competente digitalmente» es crucial, y son muchos los ciudadanos europeos que aún hoy no poseen todas las habilidades que este concepto engloba. Habría que resaltar al colectivo de profesionales de las TIC y al ámbito educativo,

\footnotetext{
${ }^{4}$ http://ec.europa.eu/social/main.jsp?catld=822\&langld=en
}

pero también al cómputo de ciudadanos que desean ser activos en la sociedad, como los grupos-objetivo que deberían beneficiarse de las medidas adoptadas en esta dirección.

De acuerdo con este marco, y frente a la realidad descrita, nace el Proyecto EPBE, con la misión de romper la brecha digital para aumentar la empleabilidad de los jóvenes europeos, mejorando sus competencias en búsqueda de empleo a través de la red y favoreciendo la creación de una marca personal online.

\section{OBJETIVOS DEL PROYEC- TO EPBE}

El Proyecto EPBE, desarrollado desde septiembre de 2015 a septiembre de 2017, tiene origen en la UDIMA, institución coordinadora del mismo. No obstante, han participado otras siete instituciones procedentes de distintos países de la Unión Europea. Dicho proyecto persigue los siguientes objetivos:

- Objetivo general. Fomentar el empleo entre los jóvenes estudiantes mediante la adquisición de competencias digitales utilizando enfoques pedagógicos innovadores y centrados en el alumno, y reforzando la integración digital en el aprendizaje.

\section{- Objetivos específicos:}

- Sensibilizar a los estudiantes y a los formadores sobre la posibilidad de adquirir competencias en las TIC para enseñar y estudiar técnicas relacionadas con la búsqueda de empleo y la marca personal en internet.

- Crear un plan de estudios multidisciplinar, basado en contextos de la vida real, para formadores y estudiantes, que involucre a diferentes sectores educativos, identificando las necesidades de formación y las competencias transversales necesarias para el desarrollo de la estrategia de aprendizaje en línea basada en la marca personal y la búsqueda de empleo.

- Compartir contenidos educativos relacionados con la marca personal y la búsqueda de empleo para aprender y enseñar mediante los recursos educativos abiertos. Apoyar la implementación de la iniciativa que partió de la Comisión Europea en 
2013 sobre la «apertura de la educación» mediante la promoción y el desarrollo de nuevos modos de aprendizaje personalizado.

- Contribuir a las reformas relacionadas con la Agenda de Modernización de la Educación Superior en Europa de 2011, la cual promueve el aprendizaje permanente a través de la creación de métodos flexibles, desarrollando modos de aumentar la tasa de empleabilidad.

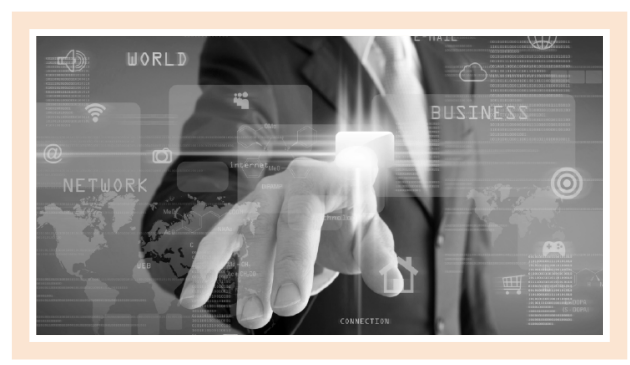

\section{DESTINATARIOS Y METODOLOGÍA}

Los destinatarios del proyecto EPBE fueron estudiantes de educación superior y formación profesional, demandantes de empleo y, en general, jóvenes de la Unión Europea, además de todos los docentes $y$ formadores pertenecientes a las instituciones participantes. También se han podido aprovechar de los productos del proyecto aquellas personas que deseasen mejorar sus referencias y aumentar su empleabilidad.

No obstante, el proyecto va mucho más allá y, por su naturaleza open, nace para hacerse extensivo y beneficiar a otros organismos o partes interesadas, como son, por ejemplo, la Administración pública y la red o comunidad internauta en general, que tienen acceso a todos los recursos educativos del proyecto. Para lograrlo, el proyecto lleva programada una serie de acciones de explotación real de los conceptos, productos y materiales generados, así como de las prestaciones «inmateriales» del mismo, que han sido y serán ejecutadas por los socios, pero también, indirectamente, por estos organismos externos.

Los socios o instituciones participantes -y a la vez beneficiarios- en el proyecto fueron:

- UDIMA (España). Universidad completamente a distancia, experta en el uso de las TIC aplicadas a la educación. Coordinadora del proyecto desde su Unidad de Innovación del Departamento de I+D+i.

- Fundació Prat (España). Fundación asociada al Centro de Estudios Prat que ofrece formación a varios niveles a través de innovadoras técnicas de enseñanza.

- Strategies and Tools to Enhance People's Skills (STePS) (Italia). Empresa pionera en enfoques formales e informales de aprendizaje para favorecer el cambio sostenible en organizaciones y territorios.
- Università degli Studi Guglielmo Marconi (Italia). Primera universidad a distancia de Italia. Dedicada a la investigación y al desarrollo y testeo de métodos pedagógicos innovadores utilizando las nuevas tecnologías.

- Marak Digital Marketing (España). Agencia de publicidad especializada en la promoción, el marketing y las tecnologías emergentes.

- Learning Plans for All (LPfA) (Grecia). Empresa experta en aprendizaje a lo largo de la vida y en proporcionar oportunidades de aprendizaje continuo, además de reunir experiencia en el campo de la formación profesional.

- Radio Leinehertz 106.5 (Alemania). Empresa sin ánimo de lucro que desempeña su labor en el campo de los medios de comunicación y en la educación de jóvenes y adultos.

- Deloitte (Chipre). Una de las empresas más grandes de Chipre, y una de las que tiene mayor crecimiento, que ofrece servicios de consultoría de todo tipo.

Los destinatarios del proyecto EPBE fueron estudiantes de educación superior y formación profesional, demandantes de empleo y, en general, jóvenes de la Unión Europea, además de todos los docentes y formadores pertenecientes a las instituciones participantes 
Figura 1. Logotipos de instituciones participantes en el proyecto EPBE

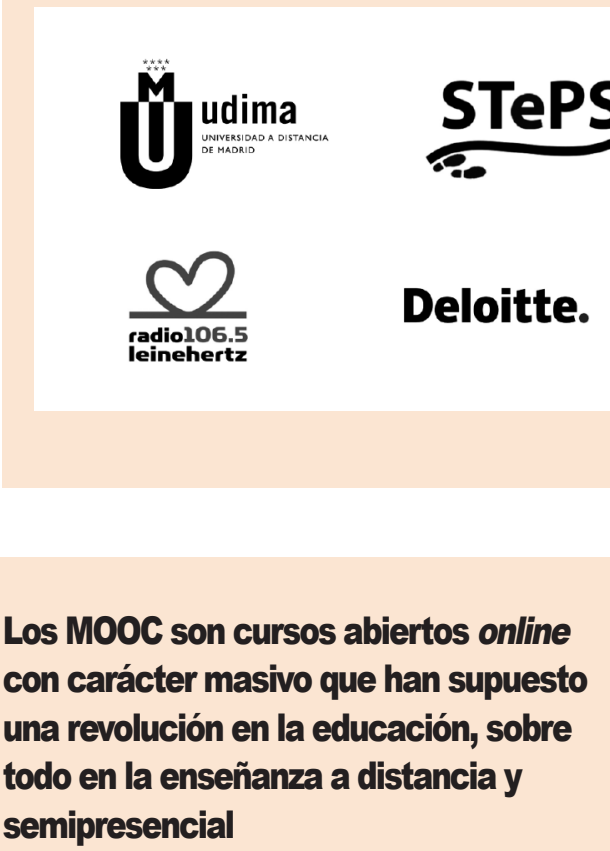

En el desarrollo e implementación del proyecto EPBE se han utilizando métodos y enfoques pedagógicos innovadores centrados en el estudiante, reforzando la integración digital en el proceso de enseñanza-aprendizaje. Además, la metodología que emplea este proyecto se plantea no solo como una innovadora manera concreta de proceder, sino como un objetivo en sí mismo. En este sentido, se utilizaron los MOOC como herramienta didáctica y como objetivo de aprendizaje a la vez. Los MOOC son cursos abiertos online con carácter masivo que han supuesto una revolución en la educación, sobre todo en la enseñanza a distancia y semipresencial.

Unido al carácter innovador, el proyecto destaca como propiedades inherentes la continuidad y la transferencia. $Y$ es que su meta consiste en que sus productos y resultados sean explotados a corto y largo plazo por otros usuarios en otros contextos y entornos, como ya hemos apuntado con anterioridad.

Haciendo alusión a las competencias digitales para la mejora de la empleabilidad, objetivo primordial de la
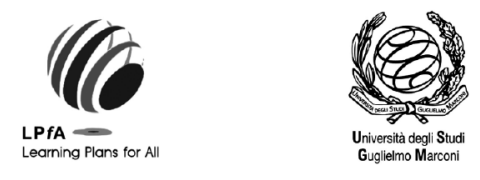

marak

Fuente: elaboración propia.

iniciativa EPBE, hay que señalar que el proyecto pone especial énfasis en el desarrollo de la marca personal y en la búsqueda de empleo 4.0, competencias que en el siglo XXI pueden considerarse imprescindibles para acceder al mercado laboral, así como para tener posibilidades de «movilidad exitosa» dentro del mismo. Para desarrollar dichas competencias digitales, las instituciones elaboraron multitud de recursos educativos abiertos, entre ellos, los citados MOOC. Estos recursos iban destinados a dos metas principalmente:

- Que los jóvenes aprendieran a implementar su marca personal a través de las redes sociales y a dominar diferentes técnicas y herramientas para la búsqueda de empleo 4.0 .

- Que los docentes y formadores adquirieran diferentes enfoques metodológicos y pedagógicos de la educación a distancia y semipresencial, para después poder formar a sus estudiantes en búsqueda de empleo y marca personal.
[...] el proyecto pone especial énfasis en el desarrollo de la marca personal y en la búsqueda de empleo 4.0, competencias que en el siglo XXI pueden considerarse imprescindibles para acceder al mercado laboral 


\section{PRODUCTOS Y RESULTADOS}

Los principales resultados generados por las instituciones participantes para la consecución de las metas anteriores fueron dos MOOC, Personal Branding (Marca Personal) y Job Hunting 4.0 (Búsqueda de Empleo 4.0), ambos disponibles gratuitamente a través de la plataforma de cursos online UDEMY.

El primero de ellos, Personal Branding, introduce el concepto de «marca personal» y ayuda al estudiante a promover exitosamente la suya propia preservando su reputación online y teniendo en cuenta todo lo referente a seguridad digital. El segundo, Job Hunting 4.0, busca que el alumno saque el máximo partido a internet y a las redes sociales en la búsqueda de empleo mediante el uso de recursos tradicionales y en línea.
Los principales resultados generados por las instituciones participantes [...] fueron dos MO0C, Personal Branding (Marca Personal) y Job Hunting 4.0 (Búsqueda de Empleo 4.0), ambos disponibles gratuitamente a través de la plataforma de cursos online UDEMY

\section{Figura 2. Pantallazos de vídeos promocionales de los MOOC y sitios web de inscripción}

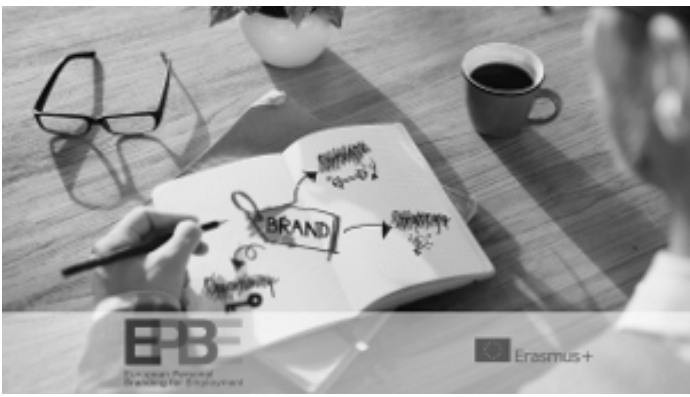

\section{MOOC Personal Branding}

Más de 1.400 personas matriculadas

(www.udemy.com/personal_branding/)

MOOC Job Hunting 4.0

Casi 1.100 personas matriculadas (www.udemy.com/job-hunting-40/)

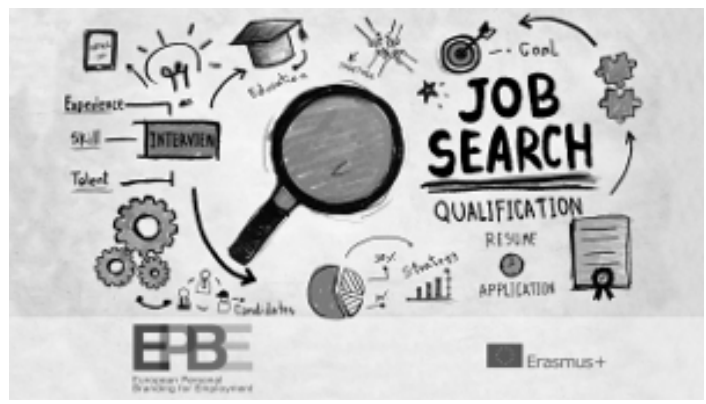

Fuente: https://youtu.be/Hvbo4cpGw8I (Personal Branding) y https://youtu.be/k99KSrmEXJ8 (Job Hunting 4.0). 


\section{Cuadro 1. Estructura de contenidos de los MOOC}

\begin{tabular}{|c|c|c|}
\hline \multicolumn{3}{|c|}{ MOOC } \\
\hline Unidad & Personal Branding & Job Hunting 4.0 \\
\hline 1 & Ideas generales sobre marca personal. & Concepto de «búsqueda de empleo $4.0 »$. \\
\hline 2 & $\begin{array}{l}\text { Habilidades de autoevaluación para una exitosa gestión de la marca } \\
\text { personal. }\end{array}$ & Concepto de «autoconocimiento» y «habilidades de autoevaluación». \\
\hline 3 & $\begin{array}{l}\text { Aspectos clave de la marca personal, establecimiento de objetivos e } \\
\text { impulso de nuestras fortalezas para lograr los primeros. }\end{array}$ & Búsqueda de empleo a través de las redes sociales. \\
\hline 4 & Concepto de «reputación online» en relación con la marca personal. & Técnicas para desarrollar un buen currículum. \\
\hline 5 & $\begin{array}{l}\text { Concepto de «marca personal» como herramienta para el desarro- } \\
\text { llo profesional. }\end{array}$ & $\begin{array}{l}\text { Consejos y recomendaciones sobre cómo abordar un proceso de } \\
\text { selección. }\end{array}$ \\
\hline
\end{tabular}

Fuente: adaptado de información publicada en http://epbe.eu/personal-branding-course/ y http://epbe.eu/job-hunting-4-0-course/.

\section{Figura 3. Pantallazo del vídeo correspondiente a la unidad 1 del MOOC Personal Branding}

$\equiv$ You Tube ${ }^{\mathrm{ES}}$

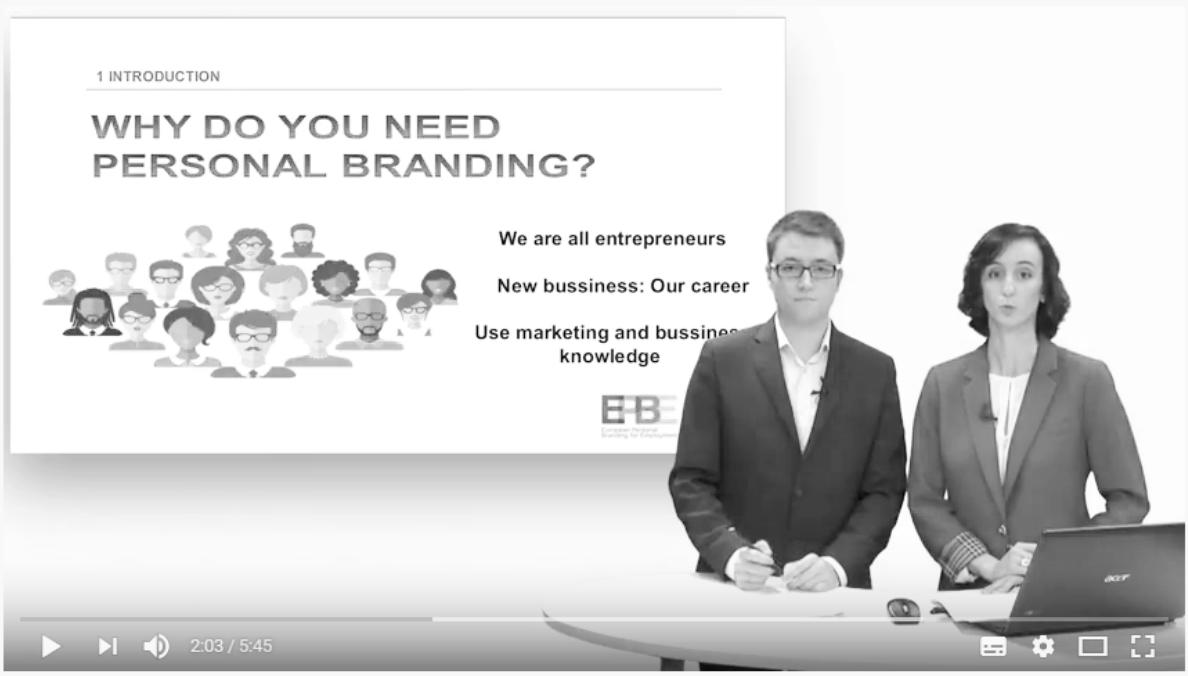

MOOC Personal Branding EPBE Unit 1: Introduction

Fuente: https://youtu.be/Bq9MvNpNbll?list=PL8NGMyit3gyfYXxxfhtPnBO3ynW2ihdMK. 


\section{Figura 4. Pantallazo del vídeo correspondiente a la unidad 3 del MOOC Job Hunting 4.0}

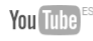

MOOC Job Hunting EPBE Unit 3: Social Media and Job Hunting

Otros resultados del proyecto han sido los siguientes:

- Repositorio de información, herramientas y recursos que están en abierto (toolkit), con los que los docentes, así como todos los que estuvieran interesados, pudieron implementar su propio MOOC.

Dentro de este recopilatorio de recursos, existen:

- Unas directrices de calidad (quality guidelines) con las que las instituciones educativas se formaron en la implementación de la marca personal y en la búsqueda de empleo 4.0. El objetivo fue asesorar a los formadores como futuros autores y profesores de los MOOC.

- Un manual de enfoques metodológicos y pedagógicos (handbook) de la educación a distancia que proporcionó los fundamentos del e-learning para que cualquiera pudiera construir y diseñar su propio curso abierto en línea (MOOC). Las sugerencias de este documento se basaron en una combinación de investigación académica y en la experiencia de cada socio del proyecto.

- Los diseños curriculares (curricular designs) de los MOOC Personal Branding y Job Hunting 4.0.
- El curso de formación Methodologies in Distance and Blended Learning (Metodologías en Enseñanza a Distancia y Semipresencial), destinado a los profesores de las instituciones participantes, cuyo fin fue capacitarles en el uso de las TIC en el aula ofreciéndoles diferentes modelos pedagógicos y metodologías, además de enseñarles cómo diseñar y desarrollar un MOOC.

\section{Cuadro 2. Componentes y sitio web del repositorio o toolkit}

\section{Toolkit}

- Quality guidelines

- Handbook

- Curricular designs

(http://epbe.eu/product/toolkit/) 


\section{- Recopilatorio de recursos educativos en abierto} (open educational resources) generados durante el proyecto para cualquiera que desease utilizarlos y que incluía documentos, folletos, vídeos, podcast, artículos académicos y resultados de interés divulgativo en general sobre marca personal y búsqueda de empleo 4.0.

Son un conjunto de recursos de los propios MOOC, así como una producción de vídeos que tratan algunos casos de buenas prácticas sobre contenidos educativos en abierto.

Para la publicación de todos los vídeos y recursos relacionados con el proyecto, UDIMA creó un canal YouTube y una página web (http://epbe.eu/).
Cuadro 3. Componentes y sitio web de los recursos educativos en abierto

\section{Open educational resources}

- Good practices

- Personal branding resources

- Job hunting 4.0 resources

(http://epbe.eu/product/oer-openeducational-resources/)

Fuente: elaboración propia.

\section{Figura 5. Pantallazo del canal YouTube del proyecto}

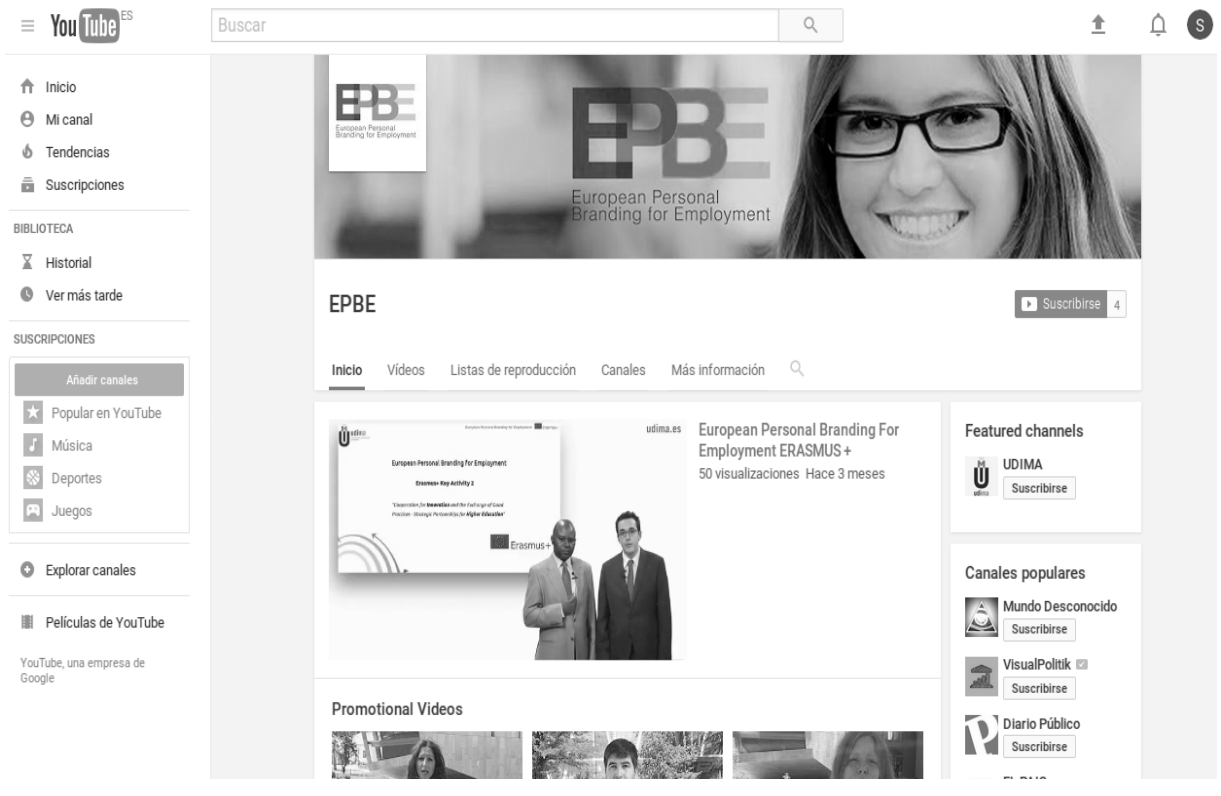

Fuente: https://www.youtube.com/channel/UCYIlo_ThkN3yZrYgnfhMQeg. 
Figura 6. Pantallazo de la web del proyecto (http:/lepbe.eu/)

\section{Welcome to EPBE Project!}

European Personal Branding for Employment (EPBE) is a project funded under the call Cooperation for innovation and the exchange of good practices - Strategic Partnerships for higher education (expedient number: 2015-1-ES01-KA203-016058).

It's main and specific objectives are aligned with the priorities as through the creation of awareness among students and trainers on the possibility of acquiring ICT skills in order to teach and study techniques related to job hunting and personal branding within Internet The project has contributed to the "development basic and transversal skills using innovative methods" with two specific contributions (project" outputs): (I) Quality Guidelines for Personal Brand Production and Job Hunting 4.0. (II) Handbook on Methodological and Pedagogical Approaches in Distance Learning for on-line personal branding and job hunting.

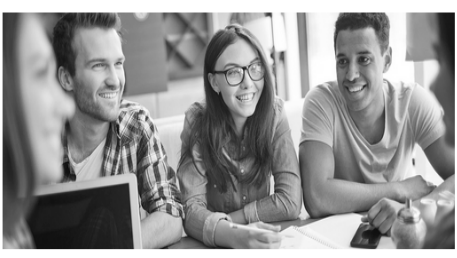

Additionally, in connection with the priority "supporting the implementation of the 2013 Communication on Opening Up Education ", EPBE has ued Open Educational Resources (OER) in order to build up an on-line personal brand and using effectively job hunting 4.0 techniques. In particular, supporting teachers, trainers, and youth

\section{CONCLUSIONES}

No cabe duda de que los resultados y los productos del proyecto aumentarán la competitividad de las instituciones, ofreciendo innovadores cursos o mejorando sus programas de enseñanza-aprendizaje con módulos que favorezcan la puesta en práctica de nuevos métodos de formación de formadores. También será beneficioso para que las instituciones académicas continúen diseñando y desarrollando cursos y metodologías innovadoras que apunten a las necesidades reales del mercado laboral.

Pero, sobre todo, los resultados cobran especial relevancia si atendemos a la reducción de desigualdades digitales entre los jóvenes que se han beneficiado del proyecto, los cuales no han resultado ser solo de
[...] los resultados y los productos del proyecto aumentarán la competitividad de las instituciones, ofreciendo innovadores cursos o mejorando sus programas de enseñanza-aprendizaje con módulos que favorezcan la puesta en práctica de nuevos métodos de formación de formadores 
Europa, sino también de Asia, África y América. Es decir, los recursos educativos abiertos, haciendo honor a su denominación, han llegado a personas de otros continentes, lo cual supone un impacto a escala mundial.

Como consecuencia de los productos que aportó el proyecto EPBE, se dieron los siguientes efectos innovadores:

- Dirigidos a estudiantes y formadores:

- Mayor utilización del aprendizaje basado en las TIC en la formación profesional y en la educación superior y de adultos.

- Mejora de los enfoques de enseñanza para el uso de las TIC en la formación.

- Incremento de la competencia para desarrollar material de e-learning abierto.

- Incremento de la competencia para trabajar profesionalmente en los entornos de e-learning y blended learning para acompañar el proceso de aprendizaje del estudiante.

- Centrados en el abordaje práctico de las técnicas de marca personal en línea y de búsqueda de empleo y dirigidos a los profesionales en esta área:

- Creación de planes de estudio de alta calidad sobre marca personal en línea, junto con el área de e-marketing.

- Creación de cursos de aprendizaje online abiertos, flexibles y fácilmente adaptables sobre marca personal y búsqueda de empleo.

- Alineación de estos planes de estudio con los instrumentos europeos de transparencia que incluye el Sistema Europeo de Créditos para la Educación y la Formación Profesionales (ECVET).

- Implementación de los cursos de formación con 60 alumnos.

\section{VALORACIÓN DEL IMPACTO DEL PROYECTO}

A continuación se presentan algunos datos numéricos que reflejan el impacto del proyecto y sus productos:

- Número de inscritos a los cursos. El número total de matriculados en los MOOC a 12 de junio de 2017 ascendía a 2.183 personas, siendo 1.213 en el caso del MOOC Personal Branding y 970 en el del MOOC Job Hunting 4.0.

Al curso Train for Trainers, para docentes y formadores, se matricularon 75 personas, según el siguiente reparto: 43 en España, 5 en Colombia, 3 en Alemania, 20 en Italia y 4 en Grecia.

- Estadísticas de la website. 17.387 páginas vistas, 3.793 sesiones, 4,58 páginas por sesión y 3 minutos y 23 segundos de media por usuario en la visita a http://epbe.eu.

- Newsletter. Primera newsletter. 3.828 suscriptores y 632 aperturas (16,51\%); International Workshop: 295 suscriptores y 103 aperturas (34,92\%); International Workshop Reminder: 295 suscriptores y 98 aperturas (33,22\%).

- Redes sociales e internet. 1.113 seguidores en Facebook, 1.448 seguidores en Twitter, 1.223 minu- tos de visualización en YouTube ( 20 h y 23 min con una media de 1 min y 23 s por usuario y 757 visualizaciones) y 94 menciones en internet.

- Asistencia al International Workshop. 70 personas.

Para lograr el mayor impacto posible del proyecto EPBE, se llevaron a cabo las siguientes acciones:

- Se creó un plan de difusión, general y local, que implementó cada institución participante. Se planificó una estrategia de comunicación para informar, difundir y concienciar sobre los objetivos, las actividades y los resultados del proyecto.

- Se creó una página web del proyecto (http://epbe.eu), disponible desde marzo de 2016.

- Se hizo una campaña a través de las redes sociales y desde los departamentos de marketing mediante e-mail.

- Artículos en prensa y artículos científicos a lo largo de la duración del proyecto.

- Cada socio realizó actividades de difusión propias.

- Se organizaron e implementaron una serie de acciones multiplicadoras, como es el repertorio online 
de recursos educativos en abierto y el International Workshop (véase figura 7), que tuvo lugar en BarceIona el 1 de junio de 2017, encuentro en el que se promovieron los citados resultados entre los socios del proyecto, los grupos-objetivo y toda aquella persona atraída por el proyecto. Entre las partes interesadas, se invitó a las instituciones regionales y nacionales locales a participar en los eventos.

Para asegurar la correcta gestión del proyecto y la calidad de los resultados, el socio Deloitte elaboró un
Plan de Gestión de la Calidad que aseguró que los procedimientos seguidos por todos los socios integrantes fueran eficaces.

Dado que el proyecto acaba de finalizar, aún no podemos entrar a valorar todas las repercusiones e impactos, tanto a nivel interno como externo, que tendrá en los próximos meses, durante los cuales se desarrollarán las acciones contempladas en el Plan de Explotación, las cuales pretenden asegurar la sostenibilidad de los resultados del proyecto una vez que este haya finalizado.

\section{Figura 7. Programa del International Workshop}

European Personal Branding for Employment (EPBE)

2015-1-ES01-KA203-016058

International Workshop

Barcelona, Thursday June, 1st 2017

Venue: Colegio de Agentes Comerciales de Barcelona

Carrer de Casp, 130, 08013 Barcelona

Thursday June, 1st 2017

09:00-09:30 Registration

09:30-11:30 Welcome and EPBE project overview

\section{9:30-09:40}

Ms Núria Prat, Director of Centre d'estudis Prat, Ms Ana Landeta, Head of EPBE project \& Mr Paul Kidd-Hewitt, Educational Consultant/ Psychologist and Entrepreneur Coach (Moderator)

\section{9:40-10:05}

Mr Josep Francí, Director of Territory and Professional Qualification of the Chamber of Commerce of Barcelona and member of its Steering Committee

10:05-10:30

Ms Mariola Dinares, Radio and TV journalist, Social

Media manager and digital entrepreneur

10:30-11:00

MrVirginio Gallardo, Humannova Founding Partner

11:00-11:30

Mr Marc Alemany, Dircom Fundacc. Fundació Audiències de la Comunicació i la Cultura. University Professor of Communication

11:30- 12:00 Coffee break
12:00-12:30 Quality Guidelines \& Handbook presentation

Mr Juan Carmona, Universidad a Distancia de Madrid Mr Roberto Righi, Steps Srl President

12:30-13:00 Personal Branding MOOC presentation and students experience Mr Ferrán Farrás, Centre d’Estudis Prat

13:00-13:30 Job Hunting 4.0 MOOC presentation and students experience Ms Vassiliki Tsekoura, Learning Plans for All Ms Angeliki Giannakopoulou, Learning Plans for All

13:30-14.00 Train for Trainers

Ms Susanna Correnti, Università degli Studi Guglielmo Marconi

14:00-14:30 Closing of the seminar

Ms Ana Landeta (moderator), Head of EPBE project Mr Pere Ros, Managing Director of Fundació BCN Formació Professional of the Town Council of Barcelona

Ms Laura Ricci, Global University In Distance Education - GUIDE Association

Mr Jesús Valdecantos, Ministry of Education, Culture and Sports

14:30-15:30 Lunch

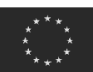

Co-funded by the

Erasmus+ Programme

of the European Union 


\section{BIBLIOGRAFÍA}

Área, M. [2010]: «¿Por qué formar en competencias informacionales y digitales en la educación superior?», Revista de Universidad y Sociedad del Conocimiento, 7 (2), Universitat Oberta de Catalunya. Disponible en: http://openaccess.uoc.edu/webapps/02/bitstream/10609/2601/1/ area-esp.pdf [Consultado: 12 de junio de 2017].

Barrenetxea, M. y Cardona, A. [2003]: «La brecha digital como fuente de nuevas desigualdades en el mercado de trabajo», Revista Economistas, 21 (95). Disponible en: https://dialnet.unirioja.es/servlet/articulo? codigo=313736 [Consultado: 12 de junio de 2017].

Comisión Europea [2006]: Recomendación del Parlamento Europeo y del Consejo sobre las competencias clave para el aprendizaje permanente. Disponible en: http://eur-lex.europa.eu/legal-content/EN/TXT/?uri= celex:32006H0962 [Consultado: 19 de junio de 2017].
[2008]: Nuevas capacidades para nuevos empleos. Previsión de las capacidades necesarias y su adecuación a las exigencias del mercado laboral. Disponible en: http://eur-lex.europa.eu/legal-content/EN/ ALL/?uri=CELEX:52008DC0868 [Consultado: 15 de junio de 2017].

[2011]: Modernización de la educación superior en la Unión Europea. Disponible en: http://eur-lex.europa. eu/legal-content/ES/TXT/?uri=celex:52011DC0567 [Consultado: 15 de junio de 2017].

[2013]: Apertura de la educación: docencia y aprendizaje innovadores para todos a través de nuevas tecnologías y recursos educativos abiertos. Disponible en: http://eur-lex.europa.eu/legal-content/EN/TX $\mathrm{T} /$ ?qid=1389115469384\&uri=CELEX:52013DC0654 [Consultado: 15 de junio de 2017].

Este máster oficial [60 créditos ECTS] se inicia en octubre y febrero de cada año y su duración normal es de 12 meses.

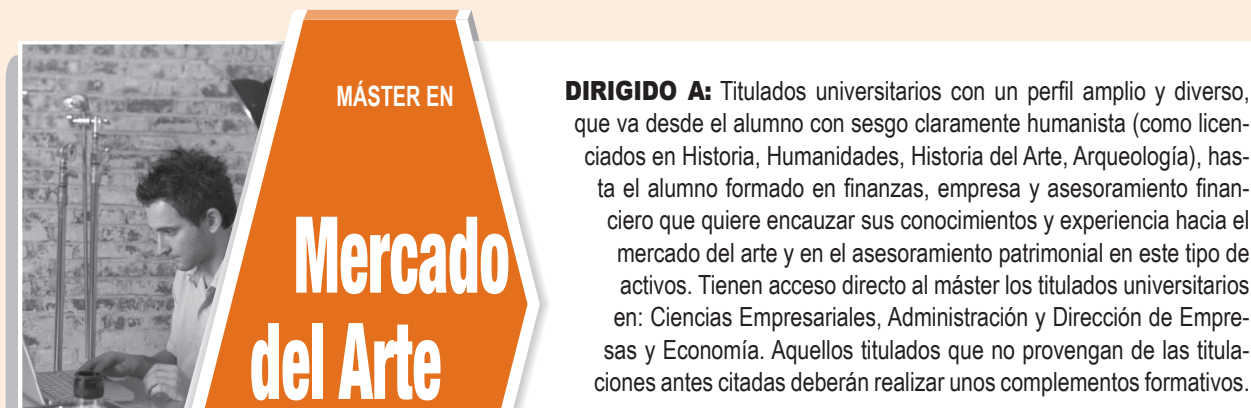

OBJETIVOS: Formar profesionales altamente cualificados en el ámbito de la dirección y gestión de empresas culturales relacionadas con el sector, dotándolos de un perfil práctico, profesional y competitivo. 